intura Fina: prevenção

\section{Projeto Cintura Fina: prevenção e controle da obesidade e demais doenças crônicas não transmissíveis}

\section{Cintura Fina Project: prevention and control of obesity and other non- comunicable chronic diseases}

\author{
Delton Manoel dos Santos Silva' \\ Raquel de Melo Vasconcelos Silva \\ Flávio Renato Barros da Guarda² \\ Silvana Gonçalves Brito de Arruda ${ }^{3}$ \\ Marina de Moraes Vasconcelos Petribú ${ }^{3}$
}

\section{RESUMO}

O projeto Cintura Fina: prevenção e controle da obesidade e demais doenças crônicas não transmissíveis (DCNT) realiza intervenções voltadas para redução da obesidade e de outras DCNT entre Usuários de Unidades Básicas de Saúde do município da Vitória de Santo Antão - PE. São executadas ações educativas interdisciplinares que fomentam a relação ensino, pesquisa e extensão e contribuem para a formação curricular dos discentes. As atividades iniciaram em março de 2012. A equipe é formada por alunos e professores dos Bacharelados em Nutrição e Educação Física do Centro Acadêmico de Vitória, além de alunos da Residência Multiprofissional das áreas de Nutrição, Educação Física, Enfermagem, Fonoaudiologia, profissionais de Educação Física e uma Enfermeira do município. As práticas ocorrem três vezes por semana. São realizadas atividade física, orientação nutricional individual e em grupo e oficinas de promoção à saúde. Os usuários participam de avaliação física e nutricional no início do programa e bimestralmente.

\section{PALAVRAS-CHAVE}

Promoção da saúde; Atividade física; Obesidade.

\begin{abstract}
The project Cintura Fina: prevention and control of obesity and other non-comunicable chronic diseases (NCCD) performs interventions aiming to reduce obesity and other NCCD among Basic Health Units users of the city of Vitoria de Santo Antão/ PE. Interdisciplinary educational activities which foster in teaching, research and extension and contribute to the training curriculum of the students are executed. The activities started in March 2012. A team formed by students and teachers of Bachelor in Nutrition and Physical Education from Centro Acadêmico de Vitória, and the Multidisciplinary Residency students in the areas of Nutrition, Physical Education, Nursing and Speech, and two Physical Education professionals and a nurse from the city. The practical activities are beld three times a week. Physical activity, individual and group nutrition counseling and workshops to promote bealth are executed. Physical and nutritional assessment is evaluated at the beginning of the program and bimonthly.
\end{abstract}

\section{KEYWORDS}

Health Promotion, Physical Activity, Obesity.
Rev Bras Ativ Fis Saúde p. 785-790 DOI

http://dx.doi.org/10.12820/rbafs.v.19n6p785

1 Núcleo de Educação Física da Universidade Federal de Pernambuco - Centro Acadêmico de Vitória

2 Centro de Pesquisa Aggeu Magalhães

3 Docente do Departamento Nutrição da Universidade Federal de Pernambuco - Centro Acadêmico de Vitória 


\section{INTRODUÇÃO}

Doenças Crônicas Não-transmissíveis (DCNT) figuram como um importante problemas de saúde pública na atualidade, sendo responsáveis por $63 \%$ da mortalidade mundial no ano de $2008^{1}$ e por $73 \%$ dos óbitos no Brasil em $2007^{2}$. Nesse contexto destaca-se a obesidade cuja expansão entre os adultos atingiu $10 \%$ da população em todas regiões do país entre 2008 e $2009^{3}$.

Tanto as DCNT, quanto a obesidade contribuem para o aumento dos gastos em saúde em diversos países ${ }^{4,5}$. Estima-se que 2004 o gasto com doenças coronarianas tenha passado dos 30 bilhões de Reais no Brasil ${ }^{6}$.

Entre os fatores de risco mais importantes para as DCNT destacam-se o tabagismo, o consumo excessivo de álcool, a alimentação inadequada e a inatividade física ${ }^{7}$.

Por outro lado, a prática de atividade física (AF), juntamente com a alimentação saudável, têm sido consideradas como importantes estratégias de prevenção e controle da obesidade e das $\mathrm{DCNT}^{8,9}$. Sua prática regular em, pelo menos, 150 minutos semanais pode reduzir os riscos de desenvolvimento dessas doenças ${ }^{10}$, promovendo efeitos sobre o sistema cardiovascular ${ }^{11}$, além de outros benefícios físicos e psicológicos ${ }^{12,13,14}$.

Nesse sentido, a Universidade Federal de Pernambuco criou em 2012 o Projeto "Cintura Fina: prevenção e controle da obesidade e demais doenças crônicas não transmissíveis".

\section{PLANEJAMENTO/ ESTRUTURACূÃO DO PROJETO}

O projeto "Cintura Fina: prevenção e controle da obesidade e demais doenças crônicas não transmissiveis" foi implantado em 2012 pelo Centro Acadêmico de Vitória/ Universidade Federal de Pernambuco (CAV/UFPE), com o objetivo de realizar gratuitamente intervenções voltadas para a redução da prevalência da obesidade e outras doenças crônicas não transmissíveis entre os usuários de Unidades de Saúde da Família (USF) do município.

O projeto foi elaborado pelo CAV/ UFPE, em parceria com a Secretaria de Saúde de Vitória de Santo Antão, visando fomentar a relação ensino, pesquisa e extensão, planejando e executando ações educativas interdisciplinares para a formação curricular dos discentes da graduação e pós graduação, a fim de que estes se identifiquem com os diversos cenários e agentes de promoção da saúde e da cidadania. O projeto visa a integração entre a Universidade e os profissionais de saúde do município, contribuindo para a melhoria dos serviços e auxiliando na obtenção de evidências que são posteriormente utilizadas em pesquisas a fim de conhecer a realidade local e auxiliar no planejamento de novas ações. O município da Vitória de Santo Antão é localizado na zona da mata Pernambucana, a $53 \mathrm{~km}$ da capital, e tem uma população de 133 mil habitantes.

O financiamento das ações é proporcionado por recursos do Programa Nacional de Reorientação da Formação Profissional em Saúde (Pró-Saúde), do Programa de Educação pelo Trabalho para a Saúde (PET-Saúde), ambos do Ministério da Saúde, além de editais da Pro-reitoria de Extensão (PROEXT) da UFPE.

A proposta está pautada nas ações do Plano Intersetorial de Prevenção e Controle da Obesidade e em consonância com a Política Nacional de Promoção da Saúde ${ }^{15}$. 
Durante a elaboração do projeto a equipe procurou identificar e elaborar ferramentas que apoiassem tanto a implantação, quanto a avaliação do Cintura Fina. Realizaram-se reuniões com professores, alunos e profissionais da secretaria de saúde e elaborou-se um modelo lógico do Projeto (figura 1).

INSUMOS

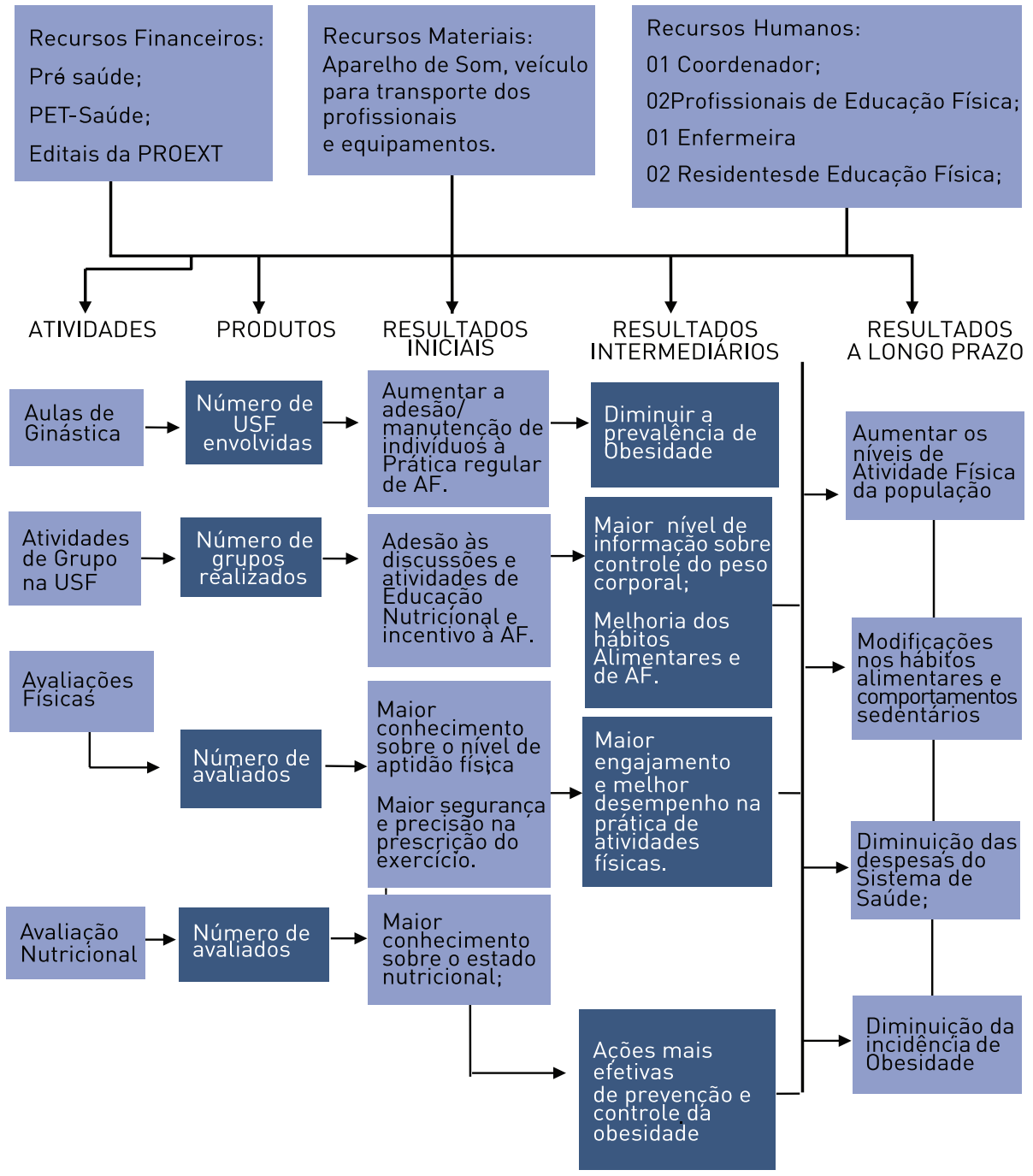

FIGURA 1 - Modelo Lógico do Projeto Cintura Fina.

\section{FUNCIONAMENTO DO PROJETO}

As atividades do Projeto iniciaram em março de 2012, pela escolha da Unidade de Saúde da Família (USF) a ser inicialmente contemplada, o planejamento das ações e a elaboração de materiais educativos. Participaram dessa etapa professores, tutores e alunos. A escolha da USF considerou o interesse demonstrado pela Equipe de Saúde da Família, e a disponibilidade de espaço físico para a realização das. Após visita a várias USF, foi escolhida a do Loteamento Conceição, que atende a aproximadamente 1200 famílias. O projeto foi elaborado para atender um máximo de 50 adultos ou idosos por USF.

A equipe do projeto é formada por alunos e professores dos Bacharelados em Nutrição e Educação Física do CAV/UFPE, residentes das áreas de Nutrição, Edu- 
cação Física, Enfermagem e Fonoaudiologia e por dois profissionais de Educação Física e uma Enfermeira do município, sendo os últimos vinculados ao Núcleo de Apoio a Saúde da Família e a USF Loteamento Conceição, respectivamente.

Inicialmente o projeto foi apresentado aos Agentes Comunitários de Saúde (ACS) da USF e foi solicitado aos mesmos que convidassem para fazer parte de uma triagem 50 indivíduos adultos ou idosos de suas micro áreas de atuação, que tivessem excesso de peso ou obesidade, além de interesse e disponibilidade para participar do projeto. Durante o período de abril a maio de 2012, foi realizada uma triagem com os 50 usuários que compareceram, por meio de avaliação nutricional (realização de medidas antropométricas), avaliação clínica (aferição da pressão arterial e glicemia capilar periférica) e física (nível de AF), além da coleta de informações socioeconômicas, demográficas e de estilo de vida (etilismo, tabagismo e hábitos alimentares). Os indivíduos de ambos os sexos, maiores 19 anos, que apresentaram excesso de peso ou obesidade (IMC $\geq 25 \mathrm{~kg} / \mathrm{m}^{2}$ para adultos, $\geq 27 \mathrm{~kg} / \mathrm{m}^{2}$ para idosos) foram encaminhados para a realização de exames cardiológicos e bioquímicos, consulta com o médico da USF e, caso necessário, com um cardiologista, a fim de obter laudo favorável para a prática regular de AF. Os exames e as consultas médicas foram realizadas nos meses de junho, julho e agosto de 2012.

No mês de setembro de 2012 iniciaram-se as atividades do projeto, com a reavaliação nutricional, avaliação física, além de uma avaliação do consumo alimentar. Dos 50 indivíduos que participaram da triagem, apenas 40 iniciaram as atividades do projeto.

Iniciou-se também a realização de atividade física três vezes por semana, com duração de 50 a 60 minutos para grupos de cerca de 20 indivíduos. Nas terçasfeiras são realizadas sessões aeróbias de ginástica, dança e circuito de exercícios. $\mathrm{Na}$ quinta-feira é realizado um trabalho de força, ginástica localizada e resistência muscular. Na sexta-feira o objetivo da sessão é trabalhar a flexibilidade, coordenação e/ou relaxamento muscular. Cada sessão é dividida em três momentos; a) uma fase de alongamento e aquecimento; b) atividades específicas para o dia, e; c) exercícios de relaxamento. Para a realização das atividades físicas o projeto dispõe de materiais tais como colchonetes, bastões, arcos, theraband, bolas de pilates, entre outros, que foram adquiridos com recursos dos editais de financiamento. A pressão arterial dos participantes é aferida no início e no final das sessões. A intensidade é monitorada por meio da escala de percepção subjetiva do esforço de Borg.

As sessões são planejadas mensalmente pela equipe da educação física do projeto, quando são definidos os dias e os tipos de atividades a serem realizadas durante o período. A equipe também se reúne duas vezes por mês, para planejar e discutir as ações e atividades com a coordenação do Projeto.

Os indivíduos inseridos no projeto recebem orientações nutricionais semanalmente por meio de consultas individuais ou atividades em grupos e orientações relacionadas à promoção da saúde e prevenção de doenças pela equipe multiprofissional do projeto. São realizadas atividades educacionais de sobre a importância da atividade física e da alimentação saudável, e ministradas dinâmicas de grupo sobre temas como obesidade e sedentarismo.

A avaliação nutricional e física é repetida bimestralmente e após 6 meses de intervenção os exames bioquímicos são reavaliados e os resultados entregues e discutidos com os usuários. Uma das limitações do projeto foi a baixa adesão dos usuários. Após 1 mês do projeto, apenas 18 usuárias frequentavam de for- 
ma assídua as atividades. O projeto permaneceu na Comunidade Loteamento Conceição por aproximadamente 1 ano e meio. Durante este período, novos usuários que se interessaram em participar do projeto e que se encaixaram nos critérios de inclusão foram inseridos e usuários que por algum motivo pessoal não pudessem mais frequentar as atividades assiduamente foram desligados.

Os discentes que participam do projeto tiveram a oportunidade de colocar em prática os conhecimentos adquiridos ao longo dos cursos de graduação, sobretudo na Atenção Básica, a qual se configura como um dos espaços de atuação profissional do egresso.

O projeto possibilitou também a realização de pesquisas, as quais originaram trabalhos apresentados em congresso e também serviu de banco de dados para a realização de três Trabalhos de Conclusão de Curso de alunos da graduação.

Observou-se até o presente momento melhora significativa no peso, índice de massa corporal e circunferência da cintura, além de melhora na autoestima e qualidade de vida dos usuários, o que demonstra que a metodologia empregada vem apresentando um resultado favorável e pode ser utilizada como base para a elaboração de políticas e estratégias públicas.

Espera-se a longo prazo uma redução na ocorrência da obesidade e outras DCNT e a diminuição dos custos diretos e indiretos com saúde no município.

Houve dificuldades para a marcação das consultas com cardiologistas, devido à demanda reprimida no município. Tal problema foi contornado através da marcação de consultas com o clínico geral da unidade de saúde, que realizava a triagem dos pacientes que realmente precisavam do cardiologista.

As equipes de saúde do município atuaram como facilitadoras no desenvolvimento das atividades e atualmente o projeto desenvolve atividades em outras USF do município.

QUADRO 1 - Atividades desenvolvidas no Projeto Cintura Fina na Comunidade Loteamento Conceição entre 2012 e 2013, Vitória de Santo Antão, PE.

\begin{tabular}{|c|c|c|c|}
\hline Atividades & Local & Periodicidade & Equipe Envolvida \\
\hline Triagem inicial & $\begin{array}{l}\text { UBS Loteamento } \\
\text { Conceição }\end{array}$ & $\begin{array}{l}\text { Abril, maio de } \\
2012\end{array}$ & $\begin{array}{l}\text { Professores, preceptores e alunos } \\
\text { de Educação Física e Nutrição }\end{array}$ \\
\hline $\begin{array}{l}\text { Avaliação física e } \\
\text { nutricional }\end{array}$ & $\begin{array}{l}\text { UBS Loteamento } \\
\text { Conceição }\end{array}$ & Mensalmente & $\begin{array}{l}\text { Professores\Residentes e alunos } \\
\text { de Educação Física e Nutrição }\end{array}$ \\
\hline Aula de ginástica & $\begin{array}{l}\text { Escola Municipal } \\
\text { do Loteamento } \\
\text { Conceição }\end{array}$ & Semanalmente & $\begin{array}{l}\text { Preceptores, Residentes e alunos } \\
\text { de Educação Física. }\end{array}$ \\
\hline $\begin{array}{l}\text { Aula de coordenação } \\
\text { e equilíbrio }\end{array}$ & $\begin{array}{l}\text { Escola Municipal } \\
\text { do Loteamento } \\
\text { Conceição }\end{array}$ & Semanalmente & $\begin{array}{l}\text { Preceptores, Residentes e alunos } \\
\text { de Educação Física. }\end{array}$ \\
\hline Aula de dança & $\begin{array}{l}\text { Escola Municipal } \\
\text { do Loteamento } \\
\text { Conceição }\end{array}$ & Semanalmente & $\begin{array}{l}\text { Professores\Residentes e alunos } \\
\text { de Educação Física }\end{array}$ \\
\hline Caminhada orientada & $\begin{array}{l}\text { Nas ruas do } \\
\text { Loteamento } \\
\text { Conceição }\end{array}$ & Semanalmente & $\begin{array}{l}\text { Residentes e alunos de Educação } \\
\text { Física. }\end{array}$ \\
\hline $\begin{array}{l}\text { Atividades educativas } \\
\text { sobre atividade física }\end{array}$ & $\begin{array}{l}\text { UBS Loteamento } \\
\text { Conceição }\end{array}$ & Semanalmente & $\begin{array}{l}\text { Professores\Residentes e alunos } \\
\text { de Educação Física }\end{array}$ \\
\hline $\begin{array}{l}\text { Aula de resistência } \\
\text { muscular }\end{array}$ & $\begin{array}{l}\text { Escola Municipal } \\
\text { do Loteamento } \\
\text { Conceição }\end{array}$ & Semanalmente & $\begin{array}{l}\text { Residentes e alunos de Educação } \\
\text { Física. }\end{array}$ \\
\hline $\begin{array}{l}\text { Orientação nutricional } \\
\text { individual lgrupo }\end{array}$ & $\begin{array}{l}\text { UBS Loteamento } \\
\text { Conceição }\end{array}$ & Semanalmente & $\begin{array}{l}\text { Professores, Preceptores e alunos } \\
\text { de Nutrição. }\end{array}$ \\
\hline
\end{tabular}




\section{REFERÊNCIAS}

1. WHO. World Health Organization. Noncommunicable diseases country profiles 2011. Geneva: WHO, 2011.

2. Schmidt MI, Duncan BB, Silva GA, Menezes AM, Monteiro CA, Barreto SM, et al. Chronic non-communicable diseases in Brazil: burden and current challenges. Lancet. 2011;377: 1949-61.

3. Instituto Brasileiro de Geografia e Estatística (IBGE). Pesquisa de Orçamentos Familiares 2008-2009. Antropometria e estado nutricional de crianças, adolescentes e adultos no Brasil. [Internet]. Rio de Janeiro: Instituto Brasileiro de Geografia e Estatística; 2010.

4. Trogdon JG, Finkelstein EA, Nwaise IA, et al. The economic burden of chronic cardiovascular disease for major insurers. Health promotion practice. 2007; 8(3):234-42.

5. American Diabetes Association. Economic costs of diabetes in the U.S. In 2007. Diabetes care 2008; 31(3):596-615.

6. Azambuja MI, Foppa M, Maranhao MF, Achutti AC. Economic burden of severe cardiovascular diseases in Brazil: an estimate based on secondary data. Arq bras de cardiologia 2008; 91(3):148-55, 63-71.

7. Brasil. Ministério da Saúde. Secretaria de Vigilância em Saúde. Vigitel Brasil 2011: Vigilância de Fatores de Risco e Proteção para Doenças Crônicas por Inquérito Telefônico. Ministério da Saúde, Secretaria de Vigilância em Saúde - Brasília: Ministério da Saúde, 2012.

8. Wijndaele K, Duvigneaud N, MATTON L. et. al. Muscular Strength, Aerobic Fitness, and Metabolic Syndrome Risk in Flemish Adults. Medicine e Science in Sports e Exercise. 2007; 39 (2), 233-240.

9. Pescatello LS, Blanchard BE, HEEST JLV et al. The Metabolic Syndrome and the immediate antihypertensive effects of aerobic exercise: a randomized control design. BioMedCentral Cardiovascular Disorders. 2008; 8:12.

10. ADA: American Diabetes Association Standards of medical care in diabetes-2010. Diabetes Care. 2010; 33(1):11-96;

11. Silva RS, Silva I, Silva RA, Souza L, Tomasi E. Atividade física e qualidade de vida. Ciências \& Saúde Coletiva. 2010;15:115-20.

12. Yohannes AM, Doherty P, Bundy C, Yalfani A. The long-term benefits of cardiac rehabilitation on depression, anxiety, physical activity and quality of life. J Clin Nurs. 2010;19:2806-13.

13. Hu G, Tuomilehto J, Borodulin K, Jousilahti P. The joint associations of occupational, commuting, and leisure-time physical activity, and the Framingham risk score on the 10-year risk of coronary heart disease. Eur Heart J. 2007;28:492-8.

14. Costa RA, Soares, HLR, Teixeira, JAC. Benefícios da atividade física e do exercício físico na depressão. Rev. Dep. Psicol. UFF. 2007; 19(1).

15. Brasil. Ministério da Saúde. Secretaria de Vigilância em Saúde. Secretaria de Atenção à Saúde. Política Nacional de Promoção da Saúde. 3. ed. Brasília: Ministério da Saúde, 2010. 60p (Série B. Textos Básicos da Saúde) (Série Pactos pela Saúde 2006; v.7).

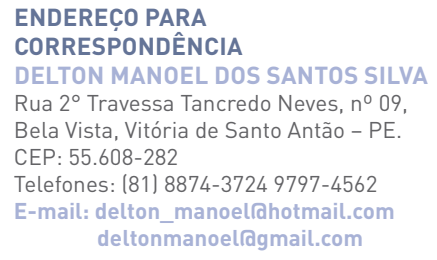

